\title{
SOLVABILITY OF SEMILINEAR EQUATIONS IN HILBERT SPACES
}

\author{
ANTONIO ACOSTA, HUGO LEIVA*
}

School of Mathematical Sciences and Information Technology, San Miguel de Urcuqui, Imbabura-100119, Ecuador

\begin{abstract}
In this paper, we study the existence of solutions for a broad class of semilinear equations in Hilbert spaces. This is done by applying the Rothe's fixed point theorem and a characterization of dense range linear operators in Hilbert spaces. As an application, we study the approximate controllability of a semilinear control system governed by a semilinear evolution equation, and a particular case of this is a control system given by a semilinear heat equation with interior control.
\end{abstract}

Keywords. Approximate controllability; Hilbert space; Semilinear equation; Semilinear evolution equation; Strongly continuous semigroup.

2010 Mathematics Subject Classification. 93B05, 93C10.

\section{INTRODUCTION}

In this paper, we study the existence of solutions for the following semilinear equation

$$
\mathscr{G}_{w}+\mathscr{H}(w)=z, \quad z \in Z, \quad w \in W
$$

where $W$ and $Z$ are Hilbert spaces, $\mathscr{G}: W \rightarrow Z$ is a bounded linear operator $(\mathscr{G} \in L(W, Z))$ and $\mathscr{H}: W \rightarrow Z$ is a nonlinear function acting as a perturbation of the following linear equation

$$
\mathscr{G}_{w}=z, \quad z \in Z, \quad w \in W .
$$

Definition 1.1. Equation (1.1) is said to be approximately solvable if, for all $z \in Z$ and $\varepsilon>0$, there exists $w \in W$ such that

$$
\left\|\mathscr{G}_{w}+\mathscr{H}(w)-z\right\|<\varepsilon .
$$

Definition 1.2. Equation (1.1) is said to be exactly solvable if, for all $z \in Z$, there exists $w \in W$ such that

$$
\mathscr{G} w+\mathscr{H}(w)=z
$$

${ }^{*}$ Corresponding author.

E-mail address: hleiva@1su.edu (H. Leiva).

Received August 29, 2017; Accepted December 21, 2017. 
Proposition 1.3. Equation (1.1) is exactly solvable (respectively approximately solvable) if the operator $\mathscr{G}+\mathscr{H}$ is surjective (respectively the operator $\mathscr{G}+\mathscr{H}$ has dense range).

Equation (1.1) has been studied in the case that $\overline{\operatorname{Rang}(\mathscr{G})}=Z$ and the nonlinear term $\mathscr{H}$ has relatively compact range; see [1] and the references therein.

The main results in this paper are as following.

Theorem 1.4. If $\overline{\operatorname{Rang}(\mathscr{G})}=Z$ and $\mathscr{H}$ is a compact mapping satisfying the condition

$$
\lim _{\|w\| \rightarrow \infty} \frac{\|\mathscr{H}(w)\|}{\|w\|}=0
$$

then equation (1.1) is approximately solvable. Moreover, for each $z \in Z$, there exists a generalized sequence $\left\{w_{\alpha}: 0 \leq \alpha \leq 1\right\}$ in $W$ such that

$$
\lim _{\alpha \rightarrow 0^{+}}\left\{\mathscr{G}_{w_{\alpha}}+\mathscr{H}\left(w_{\alpha}\right)\right\}=z
$$

where $w_{\alpha}=\mathscr{G}^{*}\left(\alpha I+\mathscr{G}^{*}\right)^{-1}\left(z-\mathscr{H}\left(w_{\alpha}\right)\right), \alpha \in(0,1]$ and the error sequence of this approximation is given by

$$
E_{\alpha} z=\alpha\left(\alpha I+\mathscr{G} \mathscr{G}^{*}\right)^{-1}\left(z-\mathscr{H}\left(w_{\alpha}\right)\right), \quad \alpha \in(0,1] .
$$

Theorem 1.5. If $\operatorname{Rang}(\mathscr{G})=Z$ and $\mathscr{H}$ is a compact mapping satisfying the condition

$$
\lim _{\|w\| \rightarrow \infty} \frac{\|\mathscr{H}(w)\|}{\|w\|}=0
$$

then equation (1.1) is exactly solvable. Moreover, for each $z \in Z$, there exists $w_{z} \in W$ such that

$$
\mathscr{G}_{w_{z}}+\mathscr{H}\left(w_{z}\right)=z
$$

where $w_{z}=\mathscr{G}^{*}\left(\mathscr{G} \mathscr{G}^{*}\right)^{-1}\left(z-\mathscr{H}\left(w_{z}\right)\right)$.

Remark 1.6. Clearly that every sub-linear function $\mathscr{H}$ satisfies condition (1.3):

$$
|\mathscr{H}(w)| \leq a_{0}\|w\|^{\eta}+b_{0}, \quad 0<\eta<1 .
$$

In this paper we will apply the following fixed point theorem to prove our main results.

Theorem 1.7. (Rothe's Fixed Theorem [2, 3, 4]) Let $E$ be a Banach space. Let $B \subset E$ be a closed convex subset such that the zero of $E$ is contained in the interior of $B$. Let $\Phi: B \rightarrow E$ be a continuous mapping with $\Phi(B)$ relatively compact in $E$ and $\Phi(\partial B) \subset B$. Then there is a point $x^{*} \in B$ such that $\Phi\left(x^{*}\right)=x^{*}$.

Finally, as an application, we shall study the controllability of the following semilinear control system

$$
\left\{\begin{array}{l}
\dot{z}=A z+B u(t)+F(t, u(t)), \quad z(t) \in Z, \quad u(t) \in U, \quad t \in(0, \tau], \\
z(0)=z_{0},
\end{array}\right.
$$

where $Z$ and $U$ are Hilbert spaces, $u \in L^{2}(0, \tau ; U), B: U \longrightarrow Z$ is a bounded linear operator, $F:[0, \tau] \times$ $U \rightarrow Z$ is smooth function, and $A: D(A) \subset Z \rightarrow Z$ is an unbounded linear operator in $Z$ which generates a strongly continuous compact semigroup $\{T(t)\}_{t \geq 0} \subset L(Z)$.

Corresponding to the semilinear system (1.4) we have the linear system

$$
\left\{\begin{array}{l}
z^{\prime}=A z+B u(t), \quad z(t) \in Z \\
z(0)=z_{0}
\end{array}\right.
$$


We assume the following hypotheses:

(A) The semigroup $\{T(t)\}_{t \geq 0}$ is compact.

(B) The linear system (1.5) is approximately controllable on $[0, \tau]$ for $\tau>0$.

(C) The function $F$ is continuous and

$$
\|F(t, u)\|_{Z} \leq a\|u\|^{\eta}+b, u \in U \quad \text { with } \quad \frac{1}{2} \leq \eta<1 .
$$

A particular case is the following control system governed by the semilinear heat equation

$$
\left\{\begin{aligned}
\frac{\partial z(t, x)}{\partial t} & =\Delta z(t, x)+1_{\omega} u(t, x)+f(t, u(t, x)), \quad t>0 \\
z(t, x) & =0, \quad \text { on } \quad(0, \tau) \times \partial \Omega \\
z(0, x) & =z_{0}(x), x \in \Omega
\end{aligned}\right.
$$

where $\Delta$ denotes the Laplacian operator, $\Omega$ is a bounded domain in $\mathbb{R}^{N}(N \geq 1), \omega$ is an open nonempty subset of $\Omega, 1_{\omega}$ denotes the characteristic function of the set $\omega$, the distributed control $u$ belongs to $L_{2}\left([0, \tau] ; L_{2}(\Omega)\right)$ and the nonlinear function $f:[0, \tau] \times \mathbb{R} \rightarrow \mathbb{R}$ is smooth enough such that

$$
|f(t, u)| \leq a_{0}|u|^{\eta}+b_{0}, \quad u \in \mathbb{R}
$$

where $\frac{1}{2} \leq \eta<1$.

The controllability of semilinear control evolution equations has been studied recently by Bashirov and Ghahramanlou [5], Bashirov and Ghahramanlou [6] and Bashirov et al. [7] using the new techniques that avoid fixed point theorems. The approximate controllability of semilinear evolution equation is also studied in $[1,8,9,10,11,12]$ recently.

\section{Solvability of LineAR EQUations}

In this section, we shall characterize the solvability of the linear equation (1.2). To this end, we note that the approximate solvability of equation (1.2) is equivalent to $\overline{\operatorname{Rang}(\mathscr{G})}=Z$ (respectively the exact solvability is equivalent to $\operatorname{Rang}(\mathscr{G})=Z$ ). In this sense we shall consider the following characterizations of dense range linear operators in Hilbert spaces.

Lemma 2.1. [1, 13, 14] Equation (1.2) is approximately solvable on if and only if one of the following statements holds:
a) $\overline{\operatorname{Rang}(\mathscr{G})}=Z$,
b) $\operatorname{Ker}\left(\mathscr{G}^{*}\right)=\{0\}$,
c) $\left\langle\mathscr{G}^{*} z, z\right\rangle>0, z \neq 0$ in $Z$,
d) $\lim _{\alpha \rightarrow 0^{+}} \alpha\left(\alpha I+\mathscr{G} \mathscr{G}^{*}\right)^{-1} z=0$.

Remark 2.2. Lemma 2.1 implies that

$$
\mathscr{G} w_{\alpha}=z-\alpha\left(\alpha I+\mathscr{G} \mathscr{G}^{*}\right)^{-1} z, \quad \forall z \in Z,
$$

where

$$
w_{\alpha}=\mathscr{G}^{*}\left(\alpha I+\mathscr{G} \mathscr{G}^{*}\right)^{-1} z, \quad \alpha \in(0,1] .
$$

So, $\lim _{\alpha \rightarrow 0} \mathscr{G} u_{\alpha}=z$ and the error $E_{\alpha} z$ of this approximation is given by

$$
E_{\alpha} z=\alpha\left(\alpha I+\mathscr{G} \mathscr{G}^{*}\right)^{-1} z, \quad \alpha \in(0,1] .
$$


Also, the family of linear operators $\Gamma_{\alpha}: Z \rightarrow W$ defined by

$$
\Gamma_{\alpha} z=\mathscr{G}^{*}\left(\alpha I+\mathscr{G} \mathscr{G}^{*}\right)^{-1} z, \quad 0<\alpha \leq 1
$$

is an approximate right inverse of the operator $\mathscr{G}$ in the sense that

$$
\lim _{\alpha \rightarrow 0} \mathscr{G} \Gamma_{\alpha}=I,
$$

in the strong topology.

Proposition 2.3. [1] If $\overline{\operatorname{Rang}(\mathscr{G})}=Z$, then

$$
\sup _{\alpha>0}\left\|\alpha\left(\alpha I+\mathscr{G} \mathscr{G}^{*}\right)^{-1}\right\| \leq 1 .
$$

Lemma 2.4. [1, 13, 14] Equation (1.2) is exactly solvable on if and only if one of the following statements holds:

a) $\operatorname{Rang}(\mathscr{G})=Z$,

b) $\exists \gamma>0$ such that $\left\langle\mathscr{G} \mathscr{G}^{*} z, z\right\rangle>\gamma\|z\|^{2}, z \in Z$,

c) $\mathscr{G}^{*}$ has right inverse.

Remark 2.5. Lemma 2.4 implies that the linear operator $\Gamma: Z \rightarrow W$ defined by

$$
\Gamma z=\mathscr{G}^{*}\left(\mathscr{G} \mathscr{G}^{*}\right)^{-1} z
$$

is an right inverse of the operator $\mathscr{G}$ in the sense that

$$
\mathscr{G} \Gamma=I .
$$

\section{Solvability of Semilinear Equations}

In this section we shall prove the main results of this paper: Theorem 1.4 and Theorem 1.5 , stated in the introduction of this paper. They are concerned with the solvability of semilinear equation (1.1).

Proof of Theorem 1.4. Motivated by Lemma 2.1 we consider for an arbitrary fixed $z \in Z$ the following family of nonlinear operators $\mathscr{K}^{\alpha}: W \rightarrow W$ defined by

$$
\mathscr{K}^{\alpha}(w)=\mathscr{G}^{*}\left(\alpha I+\mathscr{G}^{*}\right)^{-1}(z-\mathscr{H}(w)), \quad \alpha \in(0,1] .
$$

From the hypotheses of this theorem, we have that

$$
\lim _{\|w\| \rightarrow \infty} \frac{\left\|\mathscr{K}^{\alpha}(w)\right\|}{\|w\|}=0 .
$$

Claim 1. For all $\alpha \in(0,1]$, the operator $\mathscr{K}^{\alpha}$ has a fixed point. In fact, for a fixed $0<\rho<1$, there exists $R>0$ big enough such that

$$
\left\|\mathscr{K}^{\alpha}(w)\right\| \leq \rho\|w\|,\|w\|=R .
$$

Hence, if we denote by $B(0, R)$ the ball of center zero and radius $R>0$, we get that $\mathscr{K}^{\alpha}(\partial B(0, R)) \subset$ $B(0, R)$. Since $\mathscr{K}^{\alpha}$ is compact and maps the sphere $\partial B(0, R)$ into the interior of $B(0, R)$, we can apply Rothe's fixed point Theorem 1.7 to ensure the existence of a fixed point $w_{\alpha} \in B(0, R) \subset W$ such that

$$
w_{\alpha}=\mathscr{K}^{\alpha}\left(w_{\alpha}\right) .
$$


Claim 2. The generalized sequence $\left\{w_{\alpha}\right\}_{\alpha \in(0,1]}$ is bounded. In fact, for the purpose of contradiction, let us assume that $\left\{w_{\alpha}\right\}_{\alpha \in(0,1]}$ is unbounded. Then, there exits a subsequence $\left\{w_{\alpha_{n}}\right\}_{n \geq 1} \subset\left\{w_{\alpha}\right\}_{\alpha \in(0,1]}$ such that

$$
\lim _{n \rightarrow \infty}\left\|w_{\alpha_{n}}\right\|=\infty \text {. }
$$

On the other hand, from (3.1) we know, for all $\alpha \in(0,1]$, that

$$
\lim _{n \rightarrow \infty} \frac{\left\|\mathscr{K}^{\alpha}\left(w_{\alpha_{n}}\right)\right\|}{\left\|w_{\alpha_{n}}\right\|}=0
$$

Particularly, we have the following situation:

$$
\begin{aligned}
& \frac{\left\|\mathscr{K}^{\alpha_{1}}\left(u_{\alpha_{1}}\right)\right\|}{\left\|u_{\alpha_{1}}\right\|} \quad \frac{\left\|\mathscr{K}^{\alpha_{1}}\left(u_{\alpha_{2}}\right)\right\|}{\left\|u_{\alpha_{2}}\right\|} \quad \frac{\left\|\mathscr{K}^{\alpha_{1}}\left(u_{\alpha_{3}}\right)\right\|}{\left\|u_{\alpha_{3}}\right\|} \quad \ldots . . \quad \frac{\left\|\mathscr{K}^{\alpha_{1}}\left(u_{\alpha_{n}}\right)\right\|}{\left\|u_{\alpha_{n}}\right\|} \rightarrow 0, \\
& \frac{\| \mathscr{K}^{\alpha_{2}\left(u_{\alpha_{1}}\right) \|}}{\left\|u_{\alpha_{1}}\right\|} \quad \frac{\| \mathscr{K}^{\alpha_{2}\left(u_{\alpha_{2}}\right) \|}}{\left\|, u_{\alpha_{2}}\right\|} \quad \frac{\left\|\mathscr{K}^{\alpha_{2}}\left(u_{\alpha_{3}}\right)\right\|}{\left\|u_{\alpha_{3}}\right\|} \quad \ldots . . \quad \frac{\left\|\mathscr{K}^{\alpha_{2}}\left(u_{\alpha_{n}}\right)\right\|}{\left\|u_{\alpha_{n}}\right\|} \rightarrow 0,
\end{aligned}
$$

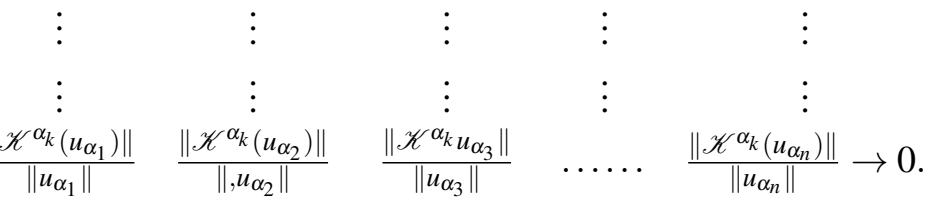

Now, applying Cantor's diagonalization process, we obtain that

$$
\lim _{n \rightarrow \infty} \frac{\left\|\mathscr{K}^{\alpha_{n}}\left(w_{\alpha_{n}}\right)\right\|}{\left\|w_{\alpha_{n}}\right\|}=0
$$

From (3.2), we arrive at

$$
\frac{\left\|\mathscr{K}^{\alpha_{n}}\left(w_{\alpha_{n}}\right)\right\|}{\left\|w_{\alpha_{n}}\right\|}=1
$$

which is a evident contradiction. Then, the claim 2 is true and there exists $\gamma>0$ such that

$$
\left\|w_{\alpha}\right\| \leq \gamma, \quad(0<\alpha \leq 1)
$$

Without loss of generality, we can assume that the generalized sequence $\mathscr{H}\left(w_{\alpha}\right)$ converges to $y \in Z$. If

$$
w_{\alpha}=\Gamma_{\alpha}\left(z-\mathscr{H}\left(w_{\alpha}\right)\right)=\mathscr{G}^{*}\left(\alpha I+\mathscr{G}^{*}\right)^{-1}\left(z-\mathscr{H}\left(w_{\alpha}\right)\right)
$$

then

$$
\begin{aligned}
\mathscr{G}_{w_{\alpha}} & =\mathscr{G} \Gamma_{\alpha}\left(z-\mathscr{H}\left(w_{\alpha}\right)\right)=\mathscr{G} \mathscr{G}^{*}\left(\alpha I+\mathscr{G} \mathscr{G}^{*}\right)^{-1}\left(z-\mathscr{H}\left(w_{\alpha}\right)\right) \\
& =\left(\alpha I+\mathscr{G} \mathscr{G}^{*}-\alpha I\right)\left(\alpha I+\mathscr{G} \mathscr{G}^{*}\right)^{-1}\left(z-\mathscr{H}\left(w_{\alpha}\right)\right) \\
& =z-\mathscr{H}\left(w_{\alpha}\right)-\alpha\left(\alpha I+\mathscr{G} \mathscr{G}^{*}\right)^{-1}\left(z-\mathscr{H}\left(w_{\alpha}\right)\right) .
\end{aligned}
$$

Hence,

$$
\mathscr{G} u_{\alpha}+\mathscr{H}\left(w_{\alpha}\right)=z-\alpha\left(\alpha I+\mathscr{G} \mathscr{G}^{*}\right)^{-1}\left(z-\mathscr{H}\left(w_{\alpha}\right)\right) .
$$

To conclude the proof of this Theorem, it enough to prove that

$$
\lim _{\alpha \rightarrow 0}\left\{-\alpha\left(\alpha I+\mathscr{G} \mathscr{G}^{*}\right)^{-1}\right\}\left(z-\mathscr{H}\left(w_{\alpha}\right)\right)=0 .
$$


From Lemma $2.1 \mathrm{~d}$ ), we get that

$$
\begin{aligned}
\lim _{\alpha \rightarrow 0}\left\{\alpha\left(\alpha I+\mathscr{G} \mathscr{G}^{*}\right)^{-1}\left(z-\mathscr{H}\left(w_{\alpha}\right)\right)\right. & =\lim _{\alpha \rightarrow 0} \alpha\left(\alpha I+\mathscr{G} \mathscr{G}^{*}\right)^{-1} y \\
& +\lim _{\alpha \rightarrow 0} \alpha\left(\alpha I+\mathscr{G} \mathscr{G}^{*}\right)^{-1}\left(\mathscr{H}\left(w_{\alpha}\right)-y\right) \\
& =\lim _{\alpha \rightarrow 0}-\alpha\left(\alpha I+\mathscr{G} \mathscr{G}^{*}\right)^{-1}\left(\mathscr{H}\left(w_{\alpha}\right)-y\right) .
\end{aligned}
$$

On the other hand, we get from Proposition 2.3 that

$$
\left\|\alpha\left(\alpha I+\mathscr{G} \mathscr{G}^{*}\right)^{-1}\left(\mathscr{H}\left(w_{\alpha}\right)-y\right)\right\| \leq\left\|\mathscr{H}\left(w_{\alpha}\right)-y\right\| .
$$

Since $\mathscr{H}\left(w_{\alpha}\right)$ converges to $y$, we get that

$$
\lim _{\alpha \rightarrow 0}\left\{-\alpha\left(\alpha I+\mathscr{G} \mathscr{G}^{*}\right)^{-1}\left(\mathscr{H}\left(w_{\alpha}\right)-y\right)\right\}=0 .
$$

Consequently,

$$
\lim _{\alpha \rightarrow 0}\left\{-\alpha\left(\alpha I+\mathscr{G} \mathscr{G}^{*}\right)^{-1} \mathscr{H}\left(w_{\alpha}\right)\right\}=0 .
$$

It follows that

$$
\lim _{\alpha \rightarrow 0}\left\{\mathscr{G}_{w_{\alpha}}+\mathscr{H}\left(w_{\alpha}\right)\right\}=z
$$

This completes the proof.

Proof of Theorem 1.5. Since $\operatorname{Rang}(\mathscr{G})=Z$, we shall apply Lemma 2.4 part c) and define for fixed $z \in Z$ the operator

$$
\mathscr{K}(w)=\mathscr{G}^{*}\left(\mathscr{G} \mathscr{G}^{*}\right)^{-1}(z-\mathscr{H}(w)), \quad w \in W .
$$

Clearly that $\mathscr{K}$ is a compact mapping and satisfies

$$
\lim _{\|w\| \rightarrow \infty} \frac{\|\mathscr{K}(w)\|}{\|w\|}=0 .
$$

Proceeding as in the second claim of the foregoing theorem we get the existence of $w_{z} \in W$ such that

$$
w_{z}=\Gamma\left(z-\mathscr{H}\left(w_{z}\right)\right)=\mathscr{G}^{*}\left(\mathscr{G} \mathscr{G}^{*}\right)^{-1}\left(z-\mathscr{H}\left(w_{z}\right)\right) .
$$

Then

$$
\mathscr{G}_{w_{z}}=z-\mathscr{H}\left(w_{z}\right) \Longleftrightarrow \mathscr{G}_{w_{z}}+\mathscr{H}\left(w_{z}\right)=z
$$

This completes the proof.

From the Banach Fixed Point Theorem, it is easy to prove the following theorem that will bee used to prove the next result of this paper.

Theorem 3.1. Let $H: W \rightarrow W$ be a Lipschitz function with a Lipschitz constant $h<1$ and consider $F(w)=w+H(w)$. Then $F$ is an homeomorphism whose inverse is a Lipschitz function with a Lipschitz constant $(1-h)^{-1}$.

Theorem 3.2. If $\operatorname{Rang}(\mathscr{G})=Z$ and the following condition holds

$$
\left\|\mathscr{H}\left(w_{2}\right)-\mathscr{H}\left(w_{1}\right)\right\| \leq L\left\|w_{2}-w_{1}\right\|, \quad w_{1}, w_{2} \in W,
$$

and $\left\|\mathscr{G}^{*}\left(\mathscr{G}_{\mathscr{G}^{*}}\right)^{-1}\right\| L<\rho<1$, then equation (1.1) is exactly solvable and a solution. The solution is given by

$$
w_{z}=\mathscr{G}^{*}\left(\mathscr{G} \mathscr{G}^{*}\right)^{-1}(I+\mathscr{H} \circ \Gamma)^{-1}(z)
$$

where $\Gamma=\mathscr{G}^{*}\left(\mathscr{G}_{\mathscr{G}^{*}}\right)^{-1}$. 
Proof. Define the operator $F=\mathscr{G}+\mathscr{H}: W \rightarrow W$. Then $F \circ \Gamma=I+\mathscr{H} \circ \Gamma$ and

$$
\left\|(\mathscr{H} \circ \Gamma)\left(z_{2}\right)-(\mathscr{H} \circ \Gamma)\left(z_{1}\right)\right\| \leq\|\Gamma\| L\left\|z_{2}-z_{1}\right\|, \quad \forall z_{1}, z_{2} \in Z,
$$

and $\|\Gamma\| L<\rho<1$. From Theorem 3.1, we find that $F \circ \Gamma=I+\mathscr{H} \circ \Gamma$ is a homeomorphis Lipschitizian whose inverse is a Lipschitz function with a Lipschitz constant $\frac{1}{1-\rho}$. It follows that

$$
F \circ\left(\Gamma \circ(I+\mathscr{H} \circ \Gamma)^{-1}\right)=I .
$$

Hence, $w_{z}=\left(\Gamma \circ(I+\mathscr{H} \circ \Gamma)^{-1}\right)(z)$ is an exact solution of (1.1). In fact,

$$
\mathscr{G}_{w_{z}}=(I+\mathscr{H} \circ \Gamma)^{-1}(z) \Longleftrightarrow(I+\mathscr{H} \circ \Gamma) \mathscr{G}_{w_{z}}=z \Longleftrightarrow \mathscr{G}_{w_{z}}+\mathscr{H}\left(\Gamma \mathscr{G} w_{z}\right)=z
$$

So, $\mathscr{G} w_{z}+\mathscr{H}\left(w_{z}\right)=z$. The proof is completed.

\section{An Application to Semilinear Control Systems}

In this section, we shall prove the approximate controllability of semilinear control system (1.4). To this end, for all $z_{0}$ and $u \in L^{2}(0, \tau ; U)$, the initial value problem

$$
\left\{\begin{array}{l}
z^{\prime}=A z+B u+F(t, u(t)), \quad z \in Z, \quad u \in U, \quad t \geq 0, \\
z(0)=z_{0},
\end{array}\right.
$$

admits only one mild solution $z$ given by

$$
\begin{aligned}
z(t) & =T(t) z_{0}+\int_{0}^{t} T(t-s) B u(s) d s \\
& +\int_{0}^{t} T(t-s) F(s, u(s)) d s .
\end{aligned}
$$

Definition 4.1. (Approximate Controllability) The system (1.4) is said to be approximately controllable on $[0, \tau]$ if for every $z_{0} \in Z, z^{1} \in Z, \varepsilon>0$ there exists $u \in L^{2}(0, \tau ; U)$ such that solution $z(t)$ of (1.1) corresponding to $u$ satisfies:

$$
\left\|z(\tau)-z^{1}\right\|_{Z}<\varepsilon
$$

Definition 4.2. For systems (1.4) and (1.5), we define the following operators. The controllability maps $\left.G, G_{F}: L_{2}(0, \tau ; U)\right) \rightarrow Z$ defined by

$$
\begin{gathered}
G u=\int_{0}^{\tau} T(\tau-s) B u(s) d s, u \in L_{2}(0, \tau ; U), \\
G_{F} u=\int_{0}^{\tau} T(\tau-s) B u(s) d s+\int_{0}^{\tau} T(\tau-s) F(s, u(s)) d s=G u+H(u), u \in L_{2}(0, \tau ; U),
\end{gathered}
$$

where

$$
H(u)=\int_{0}^{\tau} T(\tau-s) F(s, u(s)) d s .
$$

Proposition 4.3. The linear system (1.5) is approximately controllable on $[0, \tau]$ if and only if $\overline{\text { Range }(G)}=$ $Z$.

Proposition 4.4. The semilinear system (1.4) is approximately controllable on $[0, \tau]$ if and only if $\overline{\text { Range }\left(G_{F}\right)}=$ $Z$. 
Proposition 4.5. Let $(X, \Sigma, \mu)$ be a measure space with $\mu(X)<\infty$ and $1 \leq q<r<\infty$. Then $L_{r}(\mu) \subset$ $L_{q}(\mu)$ and

$$
\|f\|_{q} \leq \mu(X)^{\frac{r-q}{r q}}\|f\|_{r}, \quad f \in L_{r}(\mu) .
$$

Proof. The proof of this proposition follows from Theorem I.V.6 from [15] by putting $p=\frac{r}{q}>1$ and considering the relation

$$
\int_{X}\left(|f|^{q}\right)^{p} d \mu=\int_{X}|f|^{r} d \mu, \forall f \in L_{r}(\mu)
$$

Theorem 4.6. Under conditions $(A)$ and $(C)$, the semilinear system (1.4) is approximately controllable on $[0, \tau]$.

Proof. Since we assume that system (1.5) is approximately controllable on $[0, \tau]$, the operator $G$ satisfies all the statements from Lemma 2.1. On the other hand, since the semigroup is compact and the map $H$ given by (4.6) is compact, we have the following estimate:

$$
\|H(u)\| \leq \int_{0}^{\tau}\|T(\tau-s) F(s, u(s))\| d s \leq M \int_{0}^{\tau}\left(a\|u(s)\|^{\eta}+b\right) d s \leq \tilde{a} \int_{0}^{\tau}\|u(s)\|^{\eta} d s+\tilde{b} .
$$

Applying Holder inequality yields that

$$
\|H(u)\| \leq \tilde{a} \sqrt{\tau}\left(\int_{0}^{\tau}\|u(s)\|^{2 \eta} d s\right)^{\frac{1}{2}}+\tilde{b}=\tilde{a} \sqrt{\tau}\|u\|_{2 \eta}^{\eta}+\tilde{b} .
$$

Since $\frac{1}{2} \leq \eta<1$, we find that $1 \leq 2 \eta<2$. From proposition 4.5, we get that

$$
L_{2}(0, \tau ; U) \subset L_{2 \eta}(0, \tau ; U) \quad \text { and } \quad\|u\|_{2 \eta} \leq K\|u\|_{2}, \quad u \in L_{2}(0, \tau ; U) .
$$

Therefore,

$$
\|H(u)\| \leq \tilde{a} \sqrt{\tau} K^{\eta}\|u\|_{2}^{\eta}+\tilde{b}
$$

and

$$
\lim _{\|u\| \rightarrow \infty} \frac{\|H(u)\|}{\|u\|_{2}}=0 .
$$

Using Theorem 1.4, we conclude the proof immediately.

\section{Acknowledgement}

We would like to thank the two anonymous referees for their valuable suggestions and comments which led to the improvement of this article.

\section{REFERENCES}

[1] H. Leiva, N. Mererntes, J. Sanchez, A characterization of semilinear dense range operators and applications, Abst. Appl. Anal. 2013 (2013), Article ID 729093.

[2] J. Banas, K. Goebel, Measures of Noncompactness in Banach Spaces, Lecture Notes in Pure and Applied Mathematics, vol. 60. Marcel Dekker, New York, (1980).

[3] G. Isac, On the Rothe's fixed point theorem in general toplogical vector space, An. St. Univ. Ovidius Cnstanta, 12 (2004), $127 \mathrm{C} 134$.

[4] D.R. Smart, Fixed Point Theorems; Cambridge Tracts in Mathematics, No. 66. Cambridge University Press, London-New York, (1974). 
[5] A.E. Bashirov, N. Ghahramanlou, On partial approximate controllability of semilinear systems, Cogent Engineering 1 (2014), Article ID 965947.

[6] A.E. Bashirov, N. Gahramanlou, On partial S-controllability of semilinear partially observable systems, Int. J. Control 88 (2015), 969-982.

[7] A.E. Bashirov, N.I. Mahmudov, N. Semi, H. Etikan, Partial controllability concepts, Int. J. Control 80 (2007), 1-7.

[8] H. Leiva, N. Merentes, J.L. Sanchez, Approximate controllability of semilinear reaction diffusion equations, Math. Control Relat. Fields 2 (2012), 171-182.

[9] H. Leiva, N. Merentes, J.L. Sanchez, Interior controllability of the Benjamin-Bona-Mahony equation, J. Math. Appl. 33 (2010), $51 \mathrm{C} 59$.

[10] H. Leiva, N. Merentes, J.L. Sanchez, Interior controllability of the semilinear Benjamin-Bona-Mahony equation, J. Math. Anal. 35 (2012), 97-109.

[11] H. Leiva, N. Merentes, J.L. Sanchez, Approximate controllability of a semilinear heat equation, Int. J. Partial Differential Equations 2013 (2013), Article ID 424309.

[12] H. Leiva, Rothe's fixed point theorem and controllability of semilinear nonautonomous systems, Systems Control Lett. 67 (2014), 14-18.

[13] R.F. Curtain, A.J. Pritchard, Infinite dimensional linear systems theory, Lecture Notes in Control and Information Sciences, 8, Springer-Verlag, Berlin-Heidelberg-New York, (1978).

[14] R.F. Curtain, H. Zwart, An Introduction to Infinite Dimensional Linear Systems Theory, Text in Applied Mathematics, 21, Springer Verlag, New York, 1995.

[15] H. Brezis, Análisis funcional, Teoria y aplicaciones, Alianza Universidad Textos, Masson, Paris, 1983. 